\title{
Scandium Half-Metallocene-Catalyzed Syndiospecific Styrene Polymerization and Styrene-Ethylene Copolymerization: Unprecedented Incorporation of Syndiotactic Styrene-Styrene Sequences in Styrene-Ethylene Copolymers
}

Yunjie Luo, Jens Baldamus, and Zhaomin Hou*

Organometallic Chemistry Laboratory, RIKEN (The Institute of Physical and Chemical Research), Hiragana 2-1, Wako, Saitama 351-0198, Japan, and PRESTO, Japan Science and Technology Agency (JST), Japan

houz@riken.jp

\section{Experimental Section}

\section{Contents}

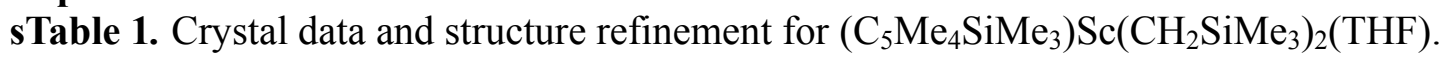

sTable 2. Atomic coordinates $\left(\mathrm{x} 10^{4}\right)$ and equivalent isotropic displacement parameters $\left(\AA^{2} \mathrm{x}\right.$ $10^{3}$ ) for $\left(\mathrm{C}_{5} \mathrm{Me}_{4} \mathrm{SiMe}_{3}\right) \mathrm{Sc}\left(\mathrm{CH}_{2} \mathrm{SiMe}_{3}\right)_{2}(\mathrm{THF})$.

sTable 3. Anisotropic displacement parameters $\left(\begin{array}{lllll}\AA^{2} & \mathrm{x} & 10^{3}\end{array}\right)$ for $\left(\mathrm{C}_{5} \mathrm{Me}_{4} \mathrm{SiMe}_{3}\right) \mathrm{Sc}\left(\mathrm{CH}_{2} \mathrm{SiMe}_{3}\right)_{2}$ (THF).

sTable 4. Bond lengths $(\AA)$ and angles $\left(^{\circ}\right)$ for $\left(\mathrm{C}_{5} \mathrm{Me}_{4} \mathrm{SiMe}_{3}\right) \mathrm{Sc}\left(\mathrm{CH}_{2} \mathrm{SiMe}_{3}\right)_{2}(\mathrm{THF})$.

sFigure $1 .{ }^{13} \mathrm{C}$ NMR spectrum of sPS.

sFigure 2. ${ }^{13} \mathrm{C}$ NMR spectra of styrene-ethylene copolymers.

sFigure 3. Aliphatic region of ${ }^{13} \mathrm{C}$ NMR spectra of styrene-ethylene copolymers.

sFigure 4. GPC curve of styrene-ethylene copolymer.

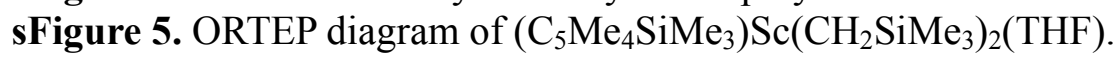

sFigure 6. DSC curve of styrene-ethylene copolymer (65\% styrene) prepared by use of $\left(\mathrm{C}_{5} \mathrm{Me}_{4} \mathrm{SiMe}_{3}\right) \mathrm{Sc}\left(\mathrm{CH}_{2} \mathrm{SiMe}_{3}\right)_{2}(\mathrm{THF}) /\left[\mathrm{Ph}_{3} \mathrm{C}\right]\left[\mathrm{B}\left(\mathrm{C}_{6} \mathrm{~F}_{5}\right)_{4}\right]$ (run 5, Table 2)

sFigure 7. DSC curve of styrene-ethylene copolymer $(87 \%$ styrene) prepared by use of $\left(\mathrm{C}_{5} \mathrm{Me}_{4} \mathrm{SiMe}_{3}\right) \mathrm{Sc}\left(\mathrm{CH}_{2} \mathrm{SiMe}_{3}\right)_{2}(\mathrm{THF}) /\left[\mathrm{Ph}_{3} \mathrm{C}\right]\left[\mathrm{B}\left(\mathrm{C}_{6} \mathrm{~F}_{5}\right)_{4}\right]$ (run 6, Table 2) 


\section{Experimental}

General Procedures and Materials. All manipulations were performed under pure argon with rigorous exclusion of air and moisture using standard Schlenk techniques and an Mbraun glovebox. Argon and ethylene (Takachiho Chemical Industrial Co., Ltd.) were purified by passing through a Dryclean column (4 A molecular sieves, Nikka Seiko Co.) and a Gasclean GC-XR column (Nikka Seiko Co.). The nitrogen in the glove box was constantly circulated through a copper/molecular sieves (4 A) catalyst unit. The oxygen and moisture in the glove box atmosphere were monitored by an $\mathrm{O}_{2} / \mathrm{H}_{2} \mathrm{O}$ Combi-Analyzer (Mbraun) to ensure the concentrations of both were always below $1 \mathrm{ppm}$. Solvents (THF, toluene, hexane) were distilled from sodium/benzophenone ketyl, degassed by the freeze-pump-thaw method, and dried over fresh $\mathrm{Na}$ chips in the glovebox. $\mathrm{LnCl}_{3}(\mathrm{Ln}=\mathrm{Sc}, \mathrm{Y}, \mathrm{Gd}, \mathrm{Lu})$ were purchased from Strem. $\mathrm{LiCH}_{2} \mathrm{SiMe}_{3}\left(1.0 \mathrm{M}\right.$ solution in pentane) and $\mathrm{C}_{5} \mathrm{Me}_{4} \mathrm{H}\left(\mathrm{SiMe}_{3}\right)$ were purchased from Aldrich, and used as received. $\left[\mathrm{Ph}_{3} \mathrm{C}\right]\left[\mathrm{B}\left(\mathrm{C}_{6} \mathrm{~F}_{5}\right)_{4}\right]$ was purchased from Tosoh Finechem Corporation and used without purification. The deuterated solvents were obtained from Cambridge Isotope. Styrene (Junsei Chemical Co., Ltd.) was dried by stirring with $\mathrm{CaH}_{2}$ for 48 hours, and distilled under reduced pressure prior to polymerization experiments.

Synthesis of $\left(\mathrm{C}_{5} \mathrm{Me}_{4} \mathrm{SiMe}_{3}\right) \mathrm{Ln}\left(\mathrm{CH}_{2} \mathrm{SiMe}_{3}\right)_{2}(\mathrm{THF})(\mathrm{Ln}=\mathrm{Sc}, \mathrm{Y}, \mathrm{Gd}$, Lu). A typical procedure for the preparation of $\left(\mathrm{C}_{5} \mathrm{Me}_{4} \mathrm{SiMe}_{3}\right) \mathrm{Sc}\left(\mathrm{CH}_{2} \mathrm{SiMe}_{3}\right)_{2}$ (THF) is given below. Other complexes can be prepared analogously. ${ }^{1}$ To a $10 \mathrm{~mL}$ of colorless hexane solution of $\mathrm{Sc}\left(\mathrm{CH}_{2} \mathrm{SiMe}_{3}\right)_{3}(\mathrm{THF})_{2}(1.366 \mathrm{~g}, 3.03 \mathrm{mmol})$, which was prepared from the reaction of $\mathrm{ScCl}_{3}$ and $\mathrm{LiCH}_{2} \mathrm{SiMe}_{3}$, ${ }^{2}$ was added $\mathrm{C}_{5} \mathrm{Me}_{4} \mathrm{H}\left(\mathrm{SiMe}_{3}\right)(0.589 \mathrm{~g}, 3.03 \mathrm{mmol})$ at room temperature. The pale yellow mixture was stirred at room temperature for 2 hours. After reduction of the solution volume under reduced pressure, the oily residue was cooled at $-30{ }^{\circ} \mathrm{C}$ overnight to give $\left(\mathrm{C}_{5} \mathrm{Me}_{4} \mathrm{SiMe}_{3}\right) \mathrm{Sc}\left(\mathrm{CH}_{2} \mathrm{SiMe}_{3}\right)_{2}$ (THF) as colorless cubic crystals (1.145 g, $2.36 \mathrm{mmol}, 78 \%$ yield $)$. ${ }^{1} \mathrm{H}$ NMR $\left(\mathrm{C}_{6} \mathrm{D}_{6}, 22{ }^{\circ} \mathrm{C}\right): \delta(\mathrm{ppm})-0.29\left(\mathrm{~d}, J_{\mathrm{H}-\mathrm{H}}=11.5 \mathrm{~Hz}, 2 \mathrm{H}, \mathrm{CH}_{2} \mathrm{SiMe}_{3},\right),-0.23\left(\mathrm{~d}, J_{\mathrm{H}-\mathrm{H}}=11.5\right.$ $\mathrm{Hz} 2 \mathrm{H}, \mathrm{CH}_{2} \mathrm{SiMe}_{3}$ ), 0.29 (s, $18 \mathrm{H}, \mathrm{CH}_{2} \mathrm{SiMe}_{3}$ ), 0.43 (s, $9 \mathrm{H}, \mathrm{C}_{5} \mathrm{Me}_{4} \mathrm{SiMe}_{3}$ ), 1.17 (br s, $4 \mathrm{H}, \mathrm{THF}$ ), $1.91\left(\mathrm{~s}, 6 \mathrm{H}, \mathrm{C}_{5} \mathrm{Me}_{4}\right), 2.22\left(\mathrm{~s}, 6 \mathrm{H}, \mathrm{C}_{5} \mathrm{Me}_{4}\right), 3.62$ (br s, $\left.4 \mathrm{H}, \mathrm{THF}\right) .{ }^{13} \mathrm{C}$ NMR $\left(\mathrm{C}_{6} \mathrm{D}_{6}, 22{ }^{\circ} \mathrm{C}\right): \delta$ (ppm) 2.83 (s, $\left.3 \mathrm{C}, \mathrm{C}_{5} \mathrm{Me}_{4} \mathrm{Si} M e_{3}\right), 4.62$ (s, $6 \mathrm{C}, \mathrm{CH}_{2} \mathrm{SiMe}$ ), 12.12 (s, $\left.2 \mathrm{C}, \mathrm{C}_{5} M e_{4}\right), 15.43$ (s, $2 \mathrm{C}$, $\mathrm{C}_{5} \mathrm{Me}_{4}$ ), 24.93 (s, $2 \mathrm{C}$, THF), 40.41 (s, $2 \mathrm{C}, \mathrm{CH}_{2} \mathrm{SiMe}_{3}$ ), 71.51 (s, $2 \mathrm{C}, \mathrm{THF}$ ), 116.64 (s, $1 \mathrm{C}$, ipso- $C_{5}\left(\mathrm{SiMe}_{3}\right) \mathrm{Me}_{4}, 124.15$ (s, 2 C, $\left.C_{5} \mathrm{Me}_{4}\right), 127.96$ (s, 2 C, $C_{5} \mathrm{Me}_{4}$ ). IR (nujol): 628 (s), 672 (s), 713 (s), 755 (s), 819 (s), 851 (s), 889 (s), 1013 (s), 1038 (m), 1131 (m), 1175 (w), 1238 (s), 1247 (s), 1280 (w), 1328 (s), 1344 (m), 1407 (w). Anal. Calcd for $\mathrm{C}_{24} \mathrm{H}_{51} \mathrm{OScSi}_{3}$ : C, 59.45; H, 10.60 . Found: C, 58.54; H, 10.41 .

${ }^{1} \mathrm{H}$ NMR Monitoring of Generation of $\left[\left(\mathrm{C}_{5} \mathrm{Me}_{4} \mathrm{SiMe}_{3}\right) \mathrm{Sc}\left(\mathrm{CH}_{2} \mathrm{SiMe}_{3}\right)(\mathrm{THF})_{\mathrm{x}}\right]\left[\mathrm{B}\left(\mathrm{C}_{6} \mathrm{~F}_{5}\right)_{4}\right]$. In a J. Young valve NMR tube, $\left(\mathrm{C}_{5} \mathrm{Me}_{4} \mathrm{SiMe}_{3}\right) \mathrm{Sc}\left(\mathrm{CH}_{2} \mathrm{SiMe}_{3}\right)_{2}$ (THF) $(10 \mathrm{mg}, 21 \mu \mathrm{mol})$ was mixed with 1 equiv of $\left[\mathrm{Ph}_{3} \mathrm{C}\right]\left[\mathrm{B}\left(\mathrm{C}_{6} \mathrm{~F}_{5}\right)_{4}\right](19 \mathrm{mg}, 21 \mu \mathrm{mol})$ in $\mathrm{C}_{6} \mathrm{D}_{6}(\mathrm{ca} .0 .8 \mathrm{~mL})$ at room temperature. A few minutes later, the ${ }^{1} \mathrm{H}$ NMR spectrum was recorded, which showed the disappearance of 1 and almost quantitative formation of $\mathrm{Ph}_{3} \mathrm{CCH}_{2} \mathrm{SiMe}_{3}(\delta 7.33-7.28(\mathrm{~m}, 6 \mathrm{H}, o-\mathrm{Ph}), \quad 7.10-7.04(\mathrm{~m}, 6 \mathrm{H}$, $m$-Ph), 7.01-6.96 (m, 3H, p-Ph), $\left.2.06\left(\mathrm{~s}, 2 \mathrm{H}, \mathrm{CH}_{2}\right),-0.21\left(\mathrm{~s}, 9 \mathrm{H}, \mathrm{SiMe}_{3}\right)\right)$. Additional signals were observed at $\delta 3.48-3.39$ (m, THF), 3.29 (br s, THF), $1.93\left(\mathrm{~s}, \mathrm{C}_{5} \mathrm{Me}_{4}\right), 1.91\left(\mathrm{~s}, \mathrm{C}_{5} \mathrm{Me}_{4}\right), 1.56(\mathrm{~s}$, $\mathrm{C}_{5} \mathrm{Me}_{4}$ ), 1.50 (s, $\mathrm{C}_{5} \mathrm{Me}_{4}$ ), 1.34-1.39 (m, THF), 1.19 (br s, THF), 0.180 (s, $\left.\mathrm{C}_{5} \mathrm{Me}_{4} \mathrm{Si}_{2} e_{3}\right),-0.06$ (s, $\left.\left.\mathrm{CH}_{2} \mathrm{SiMe}\right)_{3}\right)$, which might be assigned to $\left[\left(\mathrm{C}_{5} \mathrm{Me}_{4} \mathrm{SiMe}_{3}\right) \mathrm{Sc}\left(\mathrm{CH}_{2} \mathrm{SiMe}_{3}\right)(\mathrm{THF})_{\mathrm{x}}\right]\left[\mathrm{B}\left(\mathrm{C}_{6} \mathrm{~F}_{5}\right)_{4}\right]$, although the methylene protons in the alkyl group $\mathrm{CH}_{2} \mathrm{SiMe}_{3}$ could not be unequivocally assigned due to possible overlap with other signals. A trace amount of $\mathrm{Ph}_{3} \mathrm{CH}$ was also observed. The signals for the cationic Sc species disappeared in a few hours, as a result of decomposition to give a $\mathrm{C}_{6} \mathrm{D}_{6}$-insoluble oily product. More rapid decomposition was observed in $\mathrm{C}_{6} \mathrm{D}_{5} \mathrm{Cl}$. An 
attempt to treat the Sc cationic species with THF resulted in the polymerization of THF.

Syndiospecific Polymerization of Styrene. A typical polymerization reaction is given below (run 3, Table 1). In the glove box, a toluene solution $(7 \mathrm{~mL})$ of $\left[\mathrm{Ph}_{3} \mathrm{C}\right]\left[\mathrm{B}\left(\mathrm{C}_{6} \mathrm{~F}_{5}\right)_{4}\right](19 \mathrm{mg}, 21$ $\mu \mathrm{mol})$ was added to a toluene solution $(5 \mathrm{~mL})$ of $\left(\mathrm{C}_{5} \mathrm{Me}_{4} \mathrm{SiMe}_{3}\right) \mathrm{Sc}\left(\mathrm{CH}_{2} \mathrm{SiMe}_{3}\right)_{2}(\mathrm{THF})(10 \mathrm{mg}, 21$ $\mu \mathrm{mol})$ in a $100-\mathrm{mL}$ flask. The mixture was stirred at room temperature for a few minutes, and $2.148 \mathrm{~g}(21 \mathrm{mmol})$ of styrene was added under vigorous stirring. The magnetic stirring was ceased within a few seconds due to the viscosity. The flask was then taken outside of the glove box. After ca. $1 \mathrm{~min}$, methanol $(2 \mathrm{~mL})$ was added to terminate the polymerization. The mixture was poured into methanol $(400 \mathrm{~mL})$ to precipitate the polymer product. The white polymer powder was collected by filtration, and dried under vacuum at $60{ }^{\circ} \mathrm{C}$ to a constant weight $(2.14 \mathrm{~g}$, $100 \%)$. The polymerization in a water bath $\left(25^{\circ} \mathrm{C}\right)$ or at $-30{ }^{\circ} \mathrm{C}$ also afforded syndiotactic polystyrene similarly. The use of $\left[\mathrm{PhMe}_{2} \mathrm{NH}\right]\left[\mathrm{B}\left(\mathrm{C}_{6} \mathrm{~F}_{5}\right)_{4}\right]$ in stead of $\left[\mathrm{Ph}_{3} \mathrm{C}\right]\left[\mathrm{B}\left(\mathrm{C}_{6} \mathrm{~F}_{5}\right)_{4}\right]$ gave the similar results.

Copolymerization of Styrene with Ethylene. A typical copolymerization reaction is given below (run 4, Table 2). In the glove box, $35 \mathrm{~mL}$ of toluene, $2.148 \mathrm{~g}(21 \mathrm{mmol})$ of styrene were mixed together in a $100 \mathrm{~mL}$ two-necked flask with a stirring bar. The flask was taken outside of the glovebox and attached to a well-purged ethylene Schlenk line, and a mercury-sealed stopper. The flask was placed in a water bath at $25{ }^{\circ} \mathrm{C}$, and ethylene was then introduced under rapid stirring. The active species generated by the reaction of $21 \mu \mathrm{mol}(10 \mathrm{mg})$ of $\left(\mathrm{C}_{5} \mathrm{Me}_{4} \mathrm{SiMe}_{3}\right) \mathrm{Sc}\left(\mathrm{CH}_{2} \mathrm{SiMe}_{3}\right)_{2}$ (THF) and $21 \mu \mathrm{mol}(19 \mathrm{mg})$ of $\left[\mathrm{Ph}_{3} \mathrm{C}\right]\left[\mathrm{B}\left(\mathrm{C}_{6} \mathrm{~F}_{5}\right)_{4}\right]$ in $15 \mathrm{~mL}$ of toluene was quickly added into the flask via a syringe. The polymerization was terminated after 2 min by addition of $2 \mathrm{~mL}$ of methanol. The mixture was poured into methanol $(400 \mathrm{~mL})$ to precipitate the copolymer. The white copolymer was collected by filtration, and dried under vacuum at $60{ }^{\circ} \mathrm{C}$ to a constant weight $(0.79 \mathrm{~g})$.

Solvent Extraction. All solvent fractionations were carried out using a $100 \mathrm{~mL}$ Soxhlet extractor. $^{3}$

Homopolystyrene. Before solvent extraction, the crude polystyrenes were all characterized by NMR spectroscopic analyses for comparison. $1 \mathrm{~g}$ of polystyrene was placed in a cellulose thimble and extracted successively with $50 \mathrm{~mL}$ of boiling methyl ethyl ketone (MEK) for 4 hours. The solvent was evaporated under vacuum, and no extracts were found. The residue in the thimble was dried under vacuum at $60{ }^{\circ} \mathrm{C}$ to constant weight $(1 \mathrm{~g}, 100 \%)$, and analyzed by NMR spectroscopy. The spectra of the purified polystyrenes were identical to those of the crude polystyrenes.

Styrene-Ethylene Copolymer. A copolymer $(1 \mathrm{~g})$ was placed in a cellulose thimble and extracted successively with $50 \mathrm{~mL}$ of boiling methyl ethyl ketone (MEK) to get rid of atactic polystyrene (if any). After 4 hours, the MEK soluble fraction was dried under vacuum, and no extracts were found. Then the residue copolymer was extracted with boiling tetrahydrofuran (THF) for another 8 hours, and the copolymer was completely dissolved in boiling THF, neither insoluble PE or sPS homopolymer was isolated. The THF-soluble extract was obtained by evaporation of the solvent, dried under vacuum at $60{ }^{\circ} \mathrm{C}$, weighed (ca. $1 \mathrm{~g}$ ), and analyzed by HT-GPC, DSC, and NMR spectroscopy.

Characterization. Samples of organo rare earth metal complexes for NMR spectroscopic measurements were prepared in the glovebox using J. Young valve NMR tubes. NMR $\left({ }^{1} \mathrm{H},{ }^{13} \mathrm{C}\right)$ spectra were recorded on a JNM-EX 270 or a JNM-Alpha 600 spectrometer. IR spectra were recorded on a Shimadzu FT-IR 8100 spectrometer using nujol mulls between $\mathrm{KBr}$ disks. Elemental analyses were performed by Chemical Analysis Team, Advanced D\&S Center, RIKEN. 
${ }^{1} \mathrm{H},{ }^{13} \mathrm{C}$ NMR spectra of polymer samples were recorded on a JEOL JNM-EX 270 (FT, $300 \mathrm{MHz}$ for ${ }^{1} \mathrm{H} ; \quad 75.5 \mathrm{MHz}$ for ${ }^{13} \mathrm{C}$ ) spectrometer in 1,2-dichlorobenzene- $d_{4}$ at $130{ }^{\circ} \mathrm{C}$ or 1,1,2,2-tetrachloroethane- $d_{2}$ at $120{ }^{\circ} \mathrm{C}$. Hexamethyldisiloxane (HMDS) was used as an internal reference (2.03 ppm from TMS for ${ }^{13} \mathrm{C}$ NMR). Molecular weight and molecular weight distributions were determined against polystyrene standard by high temperature gel permeation chromatography (HT-GPC) on a HLC-8121GPC/HT apparatus (Tosoh Corporation). 1,2-Dichlorobenzene was used as an eluent at a flow rate of $1.0 \mathrm{~mL} / \mathrm{min}$ at $145{ }^{\circ} \mathrm{C}$. Melting temperatures of the resulting polymers were measured on a Perkin Elmer PYRIS Diamond DSC instrument at a heating rate of $10{ }^{\circ} \mathrm{C} / \mathrm{min}$ under helium atmosphere. Any thermal history difference in the polymers was eliminated by first heating the specimen to $300{ }^{\circ} \mathrm{C}$, cooling at 10 ${ }^{\circ} \mathrm{C} / \mathrm{min}$ to $-25^{\circ} \mathrm{C}$, and then recording the second DSC scan.

X-Ray Crystallographic Study. The crystal of $\left(\mathrm{C}_{5} \mathrm{Me}_{4} \mathrm{SiMe}_{3}\right) \mathrm{Sc}\left(\mathrm{CH}_{2} \mathrm{SiMe}_{3}\right)_{2}(\mathrm{THF})$ was sealed in a thin-walled glass capillary under a microscope in the glove box. Data collection was performed at $-40{ }^{\circ} \mathrm{C}$ on a Bruker SMART APEX diffractometer with a CCD area detector using graphite-monochromated Mo $\mathrm{K}_{\alpha}$ radiation $(\lambda=0.71069 \AA)$. The determination of crystal class and unit cell was carried out by SMART program package. ${ }^{4}$ The raw frame data were processed using $\mathrm{SAINT}^{5}$ and $\mathrm{SADABS}^{6}$ to yield the reflection data file. The structures were solved by using SHELXTL program. ${ }^{7}$ Refinement was performed on $F^{2}$ anisotropically for all the non-hydrogen atoms by the full-matrix least-squares method. The analytical scattering factors for neutral atoms were used throughout the analysis. Hydrogen atoms were placed at the calculated positions and were included in the structure calculation without further refinement of the parameters. For one of the alkyl groups disorder was found. The disordered atoms were separated in two parts (C17-C20, Si3 and C21-C24, Si4) and were treated with $80.2 \%$ and $19.8 \%$ occupancy, respectively. The residual electron densities were of no chemical significance. Crystal data and processing parameters for $\left(\mathrm{C}_{5} \mathrm{Me}_{4} \mathrm{SiMe}_{3}\right) \mathrm{Sc}\left(\mathrm{CH}_{2} \mathrm{SiMe}_{3}\right)_{2}$ (THF) are summarized in sTables 1-4, respectively.

\section{References}

(1). (a). Tardif, O.; Nishiura, M.; Hou, Z. M. Organometallics 2003, 22, 1171-1173. (b) Hultzsch,

K. C.; Spaniol, T. P.; Okuda, J. Angew. Chem. Int. Ed. 1999, 38, 227-230.

(2) Lappert, M. F.; Pearce, R. J. Chem. Soc., Chem. Comm. 1973, 126.

(3) Xu, G. X.; Lin, S. A. Macromolecules 1997, 30, 685-693.

(4). SMART Software Users Guide, version 4.21; Bruker AXS, Inc.: Madison, WI, 1997.

(5). SAINT+, Version 6.02; Bruker AXS, Inc.: Madison, WI 1999.

(6). Sheldrick, G. M. SADABS; Bruker AXS, Inc.: Madison, WI, 1998.

(7). Sheldrick, G. M. SHELXTL, Version 5.1; BUKER AXS, INC.: Madison, WI, 1998. 
sTable 1. Crystal data and structure refinement for $\left(\mathrm{C}_{5} \mathrm{Me}_{4} \mathrm{SiMe}_{3}\right) \mathrm{Sc}\left(\mathrm{CH}_{2} \mathrm{SiMe}_{3}\right)_{2}(\mathrm{THF})$

\begin{tabular}{|c|c|c|}
\hline Empirical formula & \multicolumn{2}{|l|}{ C24 H51 Sc O Si3 } \\
\hline Formula weight & \multicolumn{2}{|l|}{484.88} \\
\hline Temperature & \multicolumn{2}{|l|}{$233(2) \mathrm{K}$} \\
\hline Wavelength & \multicolumn{2}{|l|}{$0.71073 \AA$} \\
\hline Crystal system & \multicolumn{2}{|l|}{ Triclinic } \\
\hline Space group & \multicolumn{2}{|l|}{ P-1 (No. 2) } \\
\hline Unit cell dimensions & $\begin{array}{l}\mathrm{a}=10.5248(8) \AA \\
\mathrm{b}=11.7819(9) \AA \\
\mathrm{c}=12.5457(9) \AA\end{array}$ & $\begin{array}{l}\alpha=89.328(1)^{\circ} . \\
\beta=86.740(1)^{\circ} . \\
\gamma=86.326(1)^{\circ} .\end{array}$ \\
\hline Volume & $1549.9(2) \AA^{3}$ & \\
\hline $\mathrm{Z}$ & 2 & \\
\hline Density (calculated) & $1.039 \mathrm{Mg} / \mathrm{m}^{3}$ & \\
\hline $\begin{array}{l}\text { Absorption coefficient } \\
F(000)\end{array}$ & $\begin{array}{l}0.365 \mathrm{~mm}^{-1} \\
532\end{array}$ & \\
\hline Crystal size & $0.60 \times 0.50 \times 0.50$ & \\
\hline Theta range for data collection & 1.94 to $28.29^{\circ}$ & \\
\hline Index ranges & $-13<=\mathrm{h}<=13,-15<$ & $5,-16<=1<=16$ \\
\hline Reflections collected & 18609 & \\
\hline Independent reflections & $7321[\mathrm{R}$ (int) $=0.0$ & \\
\hline Completeness to theta $=28.29^{\circ}$ & $95.2 \%$ & \\
\hline Absorption correction & Semi-empirical fro & ivalents \\
\hline Max. and min. transmission & 0.8385 and 0.8106 & \\
\hline Refinement method & Full-matrix least-s & on $\mathrm{F}^{2}$ \\
\hline Data / restraints / parameters & $7321 / 2 / 324$ & \\
\hline Goodness-of-fit on $\mathrm{F}^{2}$ & 1.042 & \\
\hline Final $R$ indices $[\mathrm{I}>2 \operatorname{sigma}(\mathrm{I})]$ & $\mathrm{R} 1=0.0434, \mathrm{wR} 2$ & \\
\hline $\mathrm{R}$ indices (all data) & $\mathrm{R} 1=0.0552, \mathrm{wR} 2$ & \\
\hline Largest diff. peak and hole & 0.407 and $-0.384 \mathrm{e}$ & \\
\hline
\end{tabular}


sTable 2. Atomic coordinates $\left(\mathrm{x} 10^{4}\right)$ and equivalent isotropic displacement parameters $\left(\AA^{2} \mathrm{x}\right.$ $\left.10^{3}\right)$ for $\left(\mathrm{C}_{5} \mathrm{Me}_{4} \mathrm{SiMe}_{3}\right) \mathrm{Sc}\left(\mathrm{CH}_{2} \mathrm{SiMe}_{3}\right)_{2}$ (THF). U(eq) is defined as one third of the trace of the orthogonalized $\mathrm{Uij}^{\mathrm{ij}}$ tensor.

\begin{tabular}{|c|c|c|c|c|}
\hline & $\mathrm{x}$ & $\mathrm{y}$ & $\mathrm{z}$ & $\mathrm{U}(\mathrm{eq})$ \\
\hline $\operatorname{Sc}(1)$ & $3638(1)$ & $7728(1)$ & $2148(1)$ & $44(1)$ \\
\hline $\operatorname{Si}(1)$ & $1353(1)$ & $7432(1)$ & $-126(1)$ & $60(1)$ \\
\hline $\operatorname{Si}(2)$ & $2430(1)$ & $10566(1)$ & 2859(1) & $56(1)$ \\
\hline $\operatorname{Si}(3)$ & $2343(4)$ & $6599(2)$ & 4883(3) & $60(1)$ \\
\hline $\operatorname{Si}(4)$ & 2221(19) & $6565(13)$ & $4785(14)$ & $78(4)$ \\
\hline $\mathrm{O}(1)$ & $5454(1)$ & $8138(1)$ & $2706(1)$ & $60(1)$ \\
\hline $\mathrm{C}(1)$ & $2986(2)$ & $6017(1)$ & $1226(2)$ & $49(1)$ \\
\hline$C(2)$ & $4285(2)$ & $5774(1)$ & $1386(2)$ & $52(1)$ \\
\hline$C(3)$ & $4985(2)$ & $6553(2)$ & $768(1)$ & $51(1)$ \\
\hline $\mathrm{C}(4)$ & $4123(2)$ & $7289(2)$ & $240(1)$ & $47(1)$ \\
\hline$C(5)$ & $2854(2)$ & $6972(1)$ & $517(1)$ & $45(1)$ \\
\hline$C(6)$ & $1930(2)$ & $5311(2)$ & $1670(2)$ & $69(1)$ \\
\hline$C(7)$ & $4857(2)$ & $4797(2)$ & $2015(2)$ & $74(1)$ \\
\hline $\mathrm{C}(8)$ & $6414(2)$ & $6497(2)$ & $635(2)$ & $74(1)$ \\
\hline $\mathrm{C}(9)$ & $4503(2)$ & $8217(2)$ & $-531(2)$ & $64(1)$ \\
\hline$C(10)$ & $-21(2)$ & $7686(3)$ & $868(3)$ & $100(1)$ \\
\hline $\mathrm{C}(11)$ & $1444(3)$ & $8768(2)$ & $-928(2)$ & $93(1)$ \\
\hline$C(12)$ & $1016(3)$ & $6271(2)$ & $-1060(2)$ & $87(1)$ \\
\hline$C(13)$ & $2939(2)$ & $9527(1)$ & $1819(2)$ & $52(1)$ \\
\hline $\mathrm{C}(14)$ & 2194(3) & $12054(2)$ & $2348(2)$ & $89(1)$ \\
\hline$C(15)$ & $3624(4)$ & $10595(3)$ & 3889(3) & $132(2)$ \\
\hline$C(16)$ & $842(3)$ & $10240(3)$ & $3499(3)$ & $114(1)$ \\
\hline$C(17)$ & $2438(3)$ & $7309(2)$ & $3580(2)$ & $61(1)$ \\
\hline $\mathrm{C}(18)$ & 2941(10) & $5070(7)$ & $4788(6)$ & $125(3)$ \\
\hline C(19) & $3260(6)$ & $7312(5)$ & $5860(3)$ & $137(2)$ \\
\hline$C(20)$ & $674(4)$ & $6555(4)$ & $5451(3)$ & $103(1)$ \\
\hline$C(21)$ & $3394(11)$ & 7139(9) & $3841(6)$ & $59(3)$ \\
\hline $\mathrm{C}(22)$ & $2730(40)$ & $5020(30)$ & $5160(30)$ & $123(11)$ \\
\hline $\mathrm{C}(23)$ & $2260(20)$ & $7359(19)$ & $6162(12)$ & $112(6)$ \\
\hline $\mathrm{C}(24)$ & $524(11)$ & $6869(12)$ & $4438(13)$ & $84(4)$ \\
\hline$C(25)$ & $6087(3)$ & $7502(2)$ & $3540(2)$ & $83(1)$ \\
\hline$C(26)$ & $7434(3)$ & 7794(3) & $3417(2)$ & $94(1)$ \\
\hline$C(27)$ & $7353(3)$ & 8991(3) & 2994(3) & $92(1)$ \\
\hline $\mathrm{C}(28)$ & $6264(2)$ & $9018(2)$ & $2279(2)$ & $76(1)$ \\
\hline
\end{tabular}


sTable 3. Anisotropic displacement parameters $\left(\begin{array}{llll}\AA^{2} & \mathrm{x} & 10^{3}\end{array}\right)$ for $\left(\mathrm{C}_{5} \mathrm{Me}_{4} \mathrm{SiMe}_{3}\right) \mathrm{Sc}\left(\mathrm{CH}_{2} \mathrm{SiMe}_{3}\right)_{2}(\mathrm{THF})$. The anisotropic displacement factor exponent takes the form: $-2 p^{2}\left[h^{2} a^{*} U^{11}+2 h k a^{*} b^{*} U^{12}\right]$

\begin{tabular}{|c|c|c|c|c|c|c|}
\hline & $\mathrm{U}^{11}$ & $\mathrm{U}^{22}$ & $\mathrm{U}^{33}$ & $\mathrm{U}^{23}$ & $\mathrm{U}^{13}$ & $\mathrm{U}^{12}$ \\
\hline $\operatorname{Sc}(1)$ & $58(1)$ & $32(1)$ & $41(1)$ & $-3(1)$ & $3(1)$ & $-3(1)$ \\
\hline $\operatorname{Si}(1)$ & $55(1)$ & $54(1)$ & $71(1)$ & $-5(1)$ & $-13(1)$ & $1(1)$ \\
\hline $\operatorname{Si}(2)$ & $68(1)$ & $38(1)$ & $59(1)$ & $-8(1)$ & $9(1)$ & $1(1)$ \\
\hline $\operatorname{Si}(3)$ & $76(2)$ & $55(1)$ & $48(1)$ & $11(1)$ & $6(1)$ & $-9(1)$ \\
\hline $\operatorname{Si}(4)$ & $61(4)$ & $105(8)$ & $66(6)$ & $22(4)$ & $-1(3)$ & $-11(4)$ \\
\hline $\mathrm{O}(1)$ & $75(1)$ & $47(1)$ & $61(1)$ & $2(1)$ & $-20(1)$ & $-8(1)$ \\
\hline$C(1)$ & $58(1)$ & $34(1)$ & $55(1)$ & $-7(1)$ & $1(1)$ & $-5(1)$ \\
\hline$C(2)$ & $65(1)$ & $37(1)$ & $53(1)$ & $-10(1)$ & $-7(1)$ & $6(1)$ \\
\hline$C(3)$ & $49(1)$ & $53(1)$ & $51(1)$ & $-18(1)$ & $0(1)$ & $2(1)$ \\
\hline$C(4)$ & $54(1)$ & $45(1)$ & $42(1)$ & $-9(1)$ & $4(1)$ & $-6(1)$ \\
\hline$C(5)$ & $49(1)$ & $38(1)$ & $48(1)$ & $-6(1)$ & $-1(1)$ & $-4(1)$ \\
\hline$C(6)$ & $79(1)$ & $44(1)$ & $84(2)$ & $2(1)$ & $7(1)$ & $-17(1)$ \\
\hline$C(7)$ & $94(2)$ & $45(1)$ & $81(2)$ & $-9(1)$ & $-20(1)$ & $17(1)$ \\
\hline $\mathrm{C}(8)$ & $52(1)$ & $92(2)$ & $77(1)$ & $-28(1)$ & $0(1)$ & $7(1)$ \\
\hline C(9) & $78(1)$ & $65(1)$ & $50(1)$ & $-2(1)$ & $14(1)$ & $-16(1)$ \\
\hline$C(10)$ & $58(1)$ & $116(2)$ & $121(2)$ & $-6(2)$ & $5(2)$ & $16(1)$ \\
\hline $\mathrm{C}(11)$ & $110(2)$ & $68(2)$ & $103(2)$ & $15(1)$ & $-42(2)$ & $3(1)$ \\
\hline$C(12)$ & $87(2)$ & $77(2)$ & $103(2)$ & $-16(1)$ & $-40(2)$ & $-7(1)$ \\
\hline$C(13)$ & $63(1)$ & $39(1)$ & $53(1)$ & $-5(1)$ & $-2(1)$ & $0(1)$ \\
\hline $\mathrm{C}(14)$ & $103(2)$ & $41(1)$ & $117(2)$ & $1(1)$ & $35(2)$ & $8(1)$ \\
\hline$C(15)$ & $166(3)$ & $132(3)$ & $100(2)$ & $-62(2)$ & $-59(2)$ & $37(3)$ \\
\hline$C(16)$ & $113(2)$ & $70(2)$ & $150(3)$ & $1(2)$ & $68(2)$ & $-2(2)$ \\
\hline$C(17)$ & $80(2)$ & $49(1)$ & $52(1)$ & $6(1)$ & $10(1)$ & $4(1)$ \\
\hline$C(18)$ & $174(6)$ & $90(4)$ & $101(5)$ & $54(3)$ & $21(4)$ & $44(4)$ \\
\hline C(19) & $173(5)$ & $186(6)$ & $61(2)$ & $-1(3)$ & $-13(3)$ & $-81(5)$ \\
\hline$C(20)$ & $104(3)$ & $102(3)$ & $99(3)$ & $19(2)$ & $36(2)$ & $-6(2)$ \\
\hline $\mathrm{C}(21)$ & $57(6)$ & $55(5)$ & $64(6)$ & $8(4)$ & $8(5)$ & $-8(4)$ \\
\hline$C(22)$ & $190(20)$ & $76(12)$ & $110(20)$ & $59(15)$ & $-17(19)$ & $-31(13)$ \\
\hline$C(23)$ & $118(14)$ & $163(18)$ & $53(7)$ & $-8(9)$ & $20(8)$ & $-9(12)$ \\
\hline$C(24)$ & $52(6)$ & $83(9)$ & $116(12)$ & $8(8)$ & $7(6)$ & $-10(6)$ \\
\hline$C(25)$ & $107(2)$ & $69(1)$ & $76(2)$ & $10(1)$ & $-40(1)$ & $-7(1)$ \\
\hline$C(26)$ & $93(2)$ & $103(2)$ & $86(2)$ & $-11(2)$ & $-32(2)$ & $21(2)$ \\
\hline$C(27)$ & $72(2)$ & $106(2)$ & $101(2)$ & $-7(2)$ & $-15(1)$ & $-23(2)$ \\
\hline $\mathrm{C}(28)$ & $77(2)$ & $59(1)$ & $94(2)$ & $9(1)$ & $-12(1)$ & $-14(1)$ \\
\hline
\end{tabular}


sTable 4. Bond lengths $(\AA)$ and angles $\left(^{\circ}\right)$ for $\left(\mathrm{C}_{5} \mathrm{Me}_{4} \mathrm{SiMe}_{3}\right) \mathrm{Sc}\left(\mathrm{CH}_{2} \mathrm{SiMe}_{3}\right)_{2}(\mathrm{THF})$.

\begin{tabular}{|c|c|c|c|}
\hline $\mathrm{Sc}(1)-\mathrm{O}(1)$ & $2.1577(14)$ & $\mathrm{O}(1)-\mathrm{Sc}(1)-\mathrm{C}(5)$ & $136.78(6)$ \\
\hline $\operatorname{Sc}(1)-C(1)$ & 2.4891(17) & $\mathrm{O}(1)-\mathrm{Sc}(1)-\mathrm{C}(13)$ & $95.88(6)$ \\
\hline $\mathrm{Sc}(1)-\mathrm{C}(2)$ & $2.5406(16)$ & $\mathrm{O}(1)-\mathrm{Sc}(1)-\mathrm{C}(17)$ & $106.36(10)$ \\
\hline $\operatorname{Sc}(1)-C(3)$ & $2.5399(16)$ & $\mathrm{O}(1)-\mathrm{Sc}(1)-\mathrm{C}(21)$ & $80.5(3)$ \\
\hline $\operatorname{Sc}(1)-C(4)$ & $2.4726(16)$ & $\mathrm{C}(1)-\mathrm{Sc}(1)-\mathrm{C}(2)$ & $32.39(6)$ \\
\hline $\operatorname{Sc}(1)-C(5)$ & $2.4475(17)$ & $\mathrm{C}(1)-\mathrm{Sc}(1)-\mathrm{C}(3)$ & $53.79(6)$ \\
\hline $\operatorname{Sc}(1)-C(13)$ & $2.2402(17)$ & $\mathrm{C}(1)-\mathrm{Sc}(1)-\mathrm{C}(4)$ & $54.87(6)$ \\
\hline $\operatorname{Sc}(1)-C(17)$ & $2.206(2)$ & $\mathrm{C}(1)-\mathrm{Sc}(1)-\mathrm{C}(5)$ & $33.68(6)$ \\
\hline $\operatorname{Sc}(1)-C(21)$ & $2.233(7)$ & $\mathrm{C}(2)-\mathrm{Sc}(1)-\mathrm{C}(3)$ & $32.24(6)$ \\
\hline $\operatorname{Si}(1)-C(5)$ & $1.8611(18)$ & $\mathrm{C}(2)-\mathrm{Sc}(1)-\mathrm{C}(4)$ & $54.15(6)$ \\
\hline $\mathrm{Si}(1)-\mathrm{C}(10)$ & $1.866(3)$ & $C(2)-S c(1)-C(5)$ & $55.09(6)$ \\
\hline $\mathrm{Si}(1)-\mathrm{C}(11)$ & $1.863(3)$ & $\mathrm{C}(3)-\mathrm{Sc}(1)-\mathrm{C}(4)$ & $32.48(6)$ \\
\hline $\mathrm{Si}(1)-\mathrm{C}(12)$ & $1.876(3)$ & $\mathrm{C}(3)-\mathrm{Sc}(1)-\mathrm{C}(5)$ & $55.00(6)$ \\
\hline $\operatorname{Si}(2)-C(13)$ & $1.8333(18)$ & $\mathrm{C}(4)-\mathrm{Sc}(1)-\mathrm{C}(5)$ & $33.86(6)$ \\
\hline $\mathrm{Si}(2)-\mathrm{C}(14)$ & $1.866(2)$ & $\mathrm{C}(13)-\mathrm{Sc}(1)-\mathrm{C}(1)$ & $125.58(7)$ \\
\hline $\operatorname{Si}(2)-C(15)$ & $1.855(3)$ & $\mathrm{C}(13)-\mathrm{Sc}(1)-\mathrm{C}(2)$ & $147.01(6)$ \\
\hline $\mathrm{Si}(2)-\mathrm{C}(16)$ & $1.872(3)$ & $C(13)-\operatorname{Sc}(1)-C(3)$ & $121.86(7)$ \\
\hline $\operatorname{Si}(3)-C(17)$ & $1.827(4)$ & $\mathrm{C}(13)-\mathrm{Sc}(1)-\mathrm{C}(4)$ & $93.72(6)$ \\
\hline $\mathrm{Si}(3)-\mathrm{C}(18)$ & $1.872(9)$ & $\mathrm{C}(13)-\mathrm{Sc}(1)-\mathrm{C}(5)$ & $94.69(6)$ \\
\hline $\operatorname{Si}(3)-C(19)$ & $1.845(7)$ & $C(13)-S c(1)-C(17)$ & $101.78(8)$ \\
\hline $\operatorname{Si}(3)-C(20)$ & $1.861(5)$ & $C(13)-S c(1)-C(21)$ & $115.9(3)$ \\
\hline $\mathrm{Si}(4)-\mathrm{C}(21)$ & $1.819(9)$ & $\mathrm{C}(17)-\mathrm{Sc}(1)-\mathrm{C}(1)$ & $90.58(9)$ \\
\hline $\mathrm{Si}(4)-\mathrm{C}(22)$ & $1.93(4)$ & $\mathrm{C}(17)-\mathrm{Sc}(1)-\mathrm{C}(2)$ & $102.15(8)$ \\
\hline $\operatorname{Si}(4)-C(23)$ & $1.98(3)$ & $\mathrm{C}(17)-\mathrm{Sc}(1)-\mathrm{C}(3)$ & $134.08(8)$ \\
\hline $\mathrm{Si}(4)-\mathrm{C}(24)$ & $1.87(3)$ & $\mathrm{C}(17)-\mathrm{Sc}(1)-\mathrm{C}(4)$ & $144.45(9)$ \\
\hline $\mathrm{O}(1)-\mathrm{C}(25)$ & $1.448(3)$ & $\mathrm{C}(17)-\mathrm{Sc}(1)-\mathrm{C}(5)$ & $112.22(9)$ \\
\hline $\mathrm{O}(1)-\mathrm{C}(28)$ & $1.462(3)$ & $C(17)-S c(1)-C(21)$ & $28.2(3)$ \\
\hline$C(1)-C(2)$ & $1.404(3)$ & $\mathrm{C}(21)-\mathrm{Sc}(1)-\mathrm{C}(1)$ & $99.6(3)$ \\
\hline$C(1)-C(5)$ & $1.431(2)$ & $\mathrm{C}(21)-\mathrm{Sc}(1)-\mathrm{C}(2)$ & $95.6(3)$ \\
\hline$C(1)-C(6)$ & $1.508(3)$ & $\mathrm{C}(21)-\mathrm{Sc}(1)-\mathrm{C}(3)$ & $121.3(3)$ \\
\hline$C(2)-C(3)$ & $1.410(3)$ & $\mathrm{C}(21)-\mathrm{Sc}(1)-\mathrm{C}(4)$ & 149.7(3) \\
\hline$C(2)-C(7)$ & $1.502(3)$ & $\mathrm{C}(21)-\mathrm{Sc}(1)-\mathrm{C}(5)$ & $130.4(3)$ \\
\hline$C(3)-C(4)$ & $1.403(3)$ & $C(5)-\operatorname{Si}(1)-C(10)$ & $112.36(11)$ \\
\hline$C(3)-C(8)$ & $1.501(3)$ & $\mathrm{C}(5)-\mathrm{Si}(1)-\mathrm{C}(11)$ & $114.23(11)$ \\
\hline$C(4)-C(5)$ & $1.433(2)$ & $\mathrm{C}(5)-\mathrm{Si}(1)-\mathrm{C}(12)$ & $106.93(10)$ \\
\hline$C(4)-C(9)$ & $1.509(3)$ & $C(10)-\operatorname{Si}(1)-C(11)$ & $105.99(15)$ \\
\hline$C(25)-C(26)$ & $1.480(4)$ & $\mathrm{C}(10)-\mathrm{Si}(1)-\mathrm{C}(12)$ & $109.88(15)$ \\
\hline$C(26)-C(27)$ & $1.500(4)$ & $\mathrm{C}(11)-\mathrm{Si}(1)-\mathrm{C}(12)$ & $107.33(13)$ \\
\hline \multirow[t]{2}{*}{$\mathrm{C}(27)-\mathrm{C}(28)$} & $1.493(3)$ & $C(13)-\operatorname{Si}(2)-C(14)$ & $113.79(11)$ \\
\hline & & $C(13)-\operatorname{Si}(2)-C(15)$ & $111.10(13)$ \\
\hline $\mathrm{O}(1)-\mathrm{Sc}(1)-\mathrm{C}(1)$ & $131.19(6)$ & $C(13)-\operatorname{Si}(2)-C(16)$ & $110.72(13)$ \\
\hline $\mathrm{O}(1)-\mathrm{Sc}(1)-\mathrm{C}(2)$ & $98.80(6)$ & $C(14)-\operatorname{Si}(2)-C(15)$ & $106.38(17)$ \\
\hline $\mathrm{O}(1)-\mathrm{Sc}(1)-\mathrm{C}(3)$ & $84.21(6)$ & $C(14)-\operatorname{Si}(2)-C(16)$ & $104.68(13)$ \\
\hline $\mathrm{O}(1)-\mathrm{Sc}(1)-\mathrm{C}(4)$ & $103.59(6)$ & $C(15)-\operatorname{Si}(2)-C(16)$ & $109.9(2)$ \\
\hline
\end{tabular}




$\begin{array}{llll}\mathrm{C}(17)-\mathrm{Si}(3)-\mathrm{C}(18) & 111.5(3) & \mathrm{C}(4)-\mathrm{C}(3)-\mathrm{C}(8) & 127.16(19) \\ \mathrm{C}(17)-\mathrm{Si}(3)-\mathrm{C}(19) & 111.4(2) & \mathrm{C}(4)-\mathrm{C}(5)-\mathrm{Si}(1) & 128.36(13) \\ \mathrm{C}(17)-\mathrm{Si}(3)-\mathrm{C}(20) & 112.6(3) & \mathrm{C}(5)-\mathrm{C}(1)-\mathrm{C}(6) & 126.44(18) \\ \mathrm{C}(18)-\mathrm{Si}(3)-\mathrm{C}(19) & 108.9(5) & \mathrm{C}(5)-\mathrm{C}(4)-\mathrm{C}(9) & 126.65(18) \\ \mathrm{C}(18)-\mathrm{Si}(3)-\mathrm{C}(20) & 104.6(3) & \mathrm{C}(1)-\mathrm{C}(2)-\mathrm{Sc}(1) & 71.79(9) \\ \mathrm{C}(19)-\mathrm{Si}(3)-\mathrm{C}(20) & 107.6(3) & \mathrm{C}(1)-\mathrm{C}(5)-\mathrm{Sc}(1) & 74.75(10) \\ \mathrm{C}(21)-\mathrm{Si}(4)-\mathrm{C}(22) & 110.3(14) & \mathrm{C}(2)-\mathrm{C}(1)-\mathrm{Sc}(1) & 75.82(10) \\ \mathrm{C}(21)-\mathrm{Si}(4)-\mathrm{C}(23) & 108.9(12) & \mathrm{C}(2)-\mathrm{C}(3)-\mathrm{Sc}(1) & 73.91(10) \\ \mathrm{C}(21)-\mathrm{Si}(4)-\mathrm{C}(24) & 114.5(11) & \mathrm{C}(3)-\mathrm{C}(2)-\mathrm{Sc}(1) & 73.86(10) \\ \mathrm{C}(22)-\mathrm{Si}(4)-\mathrm{C}(23) & 102.5(16) & \mathrm{C}(3)-\mathrm{C}(4)-\mathrm{Sc}(1) & 76.40(10) \\ \mathrm{C}(22)-\mathrm{Si}(4)-\mathrm{C}(24) & 117.2(17) & \mathrm{C}(4)-\mathrm{C}(3)-\mathrm{Sc}(1) & 71.12(9) \\ \mathrm{C}(23)-\mathrm{Si}(4)-\mathrm{C}(24) & 102.0(11) & \mathrm{C}(4)-\mathrm{C}(5)-\mathrm{Sc}(1) & 74.03(9) \\ \mathrm{C}(25)-\mathrm{O}(1)-\mathrm{Sc}(1) & 123.29(14) & \mathrm{C}(5)-\mathrm{C}(1)-\mathrm{Sc}(1) & 71.56(9) \\ \mathrm{C}(25)-\mathrm{O}(1)-\mathrm{C}(28) & 109.81(17) & \mathrm{C}(5)-\mathrm{C}(4)-\mathrm{Sc}(1) & 72.11(9) \\ \mathrm{C}(28)-\mathrm{O}(1)-\mathrm{Sc}(1) & 126.79(13) & \mathrm{C}(6)-\mathrm{C}(1)-\mathrm{Sc}(1) & 122.63(13) \\ \mathrm{C}(1)-\mathrm{C}(2)-\mathrm{C}(3) & 107.91(16) & \mathrm{C}(7)-\mathrm{C}(2)-\mathrm{Sc}(1) & 124.45(13) \\ \mathrm{C}(1)-\mathrm{C}(2)-\mathrm{C}(7) & 126.79(19) & \mathrm{C}(8)-\mathrm{C}(3)-\mathrm{Sc}(1) & 125.54(13) \\ \mathrm{C}(1)-\mathrm{C}(5)-\mathrm{C}(4) & 105.93(15) & \mathrm{C}(9)-\mathrm{C}(4)-\mathrm{Sc}(1) & 120.09(12) \\ \mathrm{C}(1)-\mathrm{C}(5)-\mathrm{Si}(1) & 124.24(13) & \mathrm{Si}(1)-\mathrm{C}(5)-\mathrm{Sc}(1) & 127.11(8) \\ \mathrm{C}(2)-\mathrm{C}(1)-\mathrm{C}(5) & 109.04(16) & \mathrm{Si}(2)-\mathrm{C}(13)-\mathrm{Sc}(1) & 124.09(10) \\ \mathrm{C}(2)-\mathrm{C}(1)-\mathrm{C}(6) & 124.36(18) & \mathrm{Si}(3)-\mathrm{C}(17)-\mathrm{Sc}(1) & 147.3(2) \\ \mathrm{C}(2)-\mathrm{C}(3)-\mathrm{C}(4) & 108.44(16) & \mathrm{Si}(4)-\mathrm{C}(21)-\mathrm{Sc}(1) & 141.4(10) \\ \mathrm{C}(2)-\mathrm{C}(3)-\mathrm{C}(8) & 124.14(19) & \mathrm{O}(1)-\mathrm{C}(25)-\mathrm{C}(26) & 105.1(2) \\ \mathrm{C}(3)-\mathrm{C}(4)-\mathrm{C}(5) & 108.67(15) & \mathrm{O}(1)-\mathrm{C}(28)-\mathrm{C}(27) & 104.8(2) \\ \mathrm{C}(3)-\mathrm{C}(2)-\mathrm{C}(7) & 125.06(19) & \mathrm{C}(25)-\mathrm{C}(26)-\mathrm{C}(27) & 103.7(2) \\ \mathrm{C}(3)-\mathrm{C}(4)-\mathrm{C}(9) & 124.62(18) & \mathrm{C}(28)-\mathrm{C}(27)-\mathrm{C}(26) & 103.9(2)\end{array}$




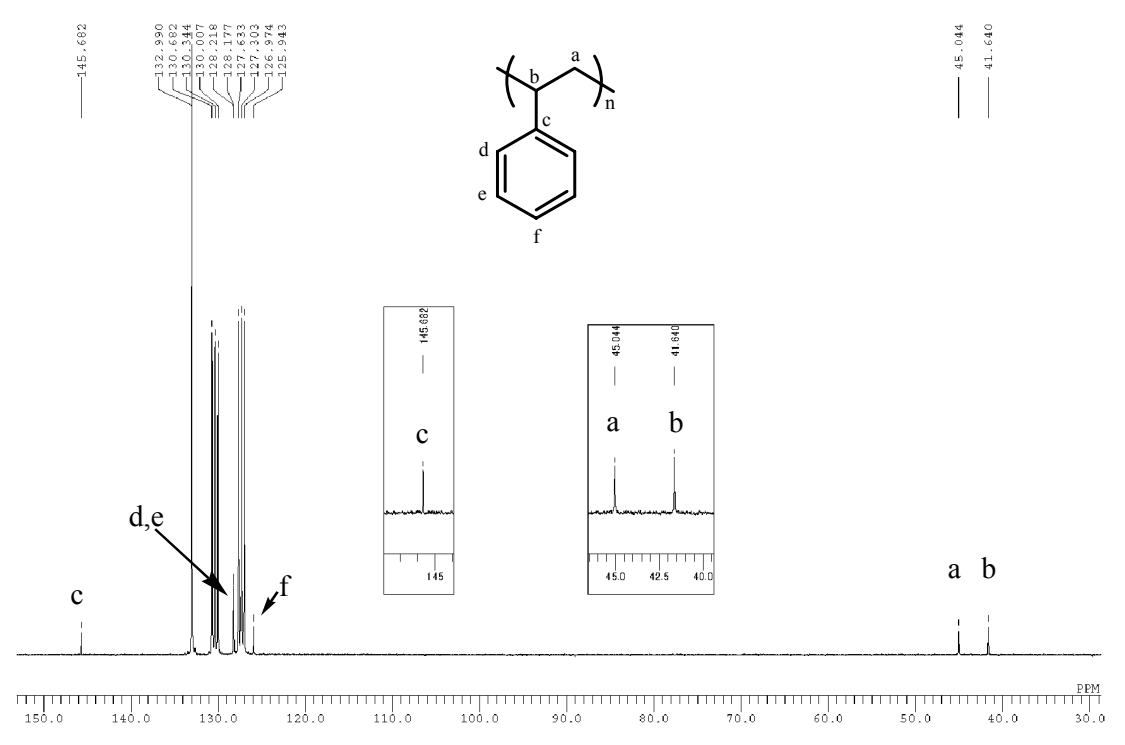

sFigure 1. $\quad{ }^{13} \mathrm{C} \quad \mathrm{NMR} \quad$ spectrum of $\quad$ SPS prepared from $\left(\mathrm{C}_{5} \mathrm{Me}_{4} \mathrm{SiMe}_{3}\right) \mathrm{Sc}\left(\mathrm{CH}_{2} \mathrm{SiMe}_{3}\right)_{2}(\mathrm{THF}) /\left[\mathrm{Ph}_{3} \mathrm{C}\right]\left[\mathrm{B}\left(\mathrm{C}_{6} \mathrm{~F}_{5}\right)_{4}\right]$ (run 3, Table 1) in 1,2-dichlorobenzene- $d_{4}$ at $130{ }^{\circ} \mathrm{C}(75.5 \mathrm{MHz}$, HMDS scale).

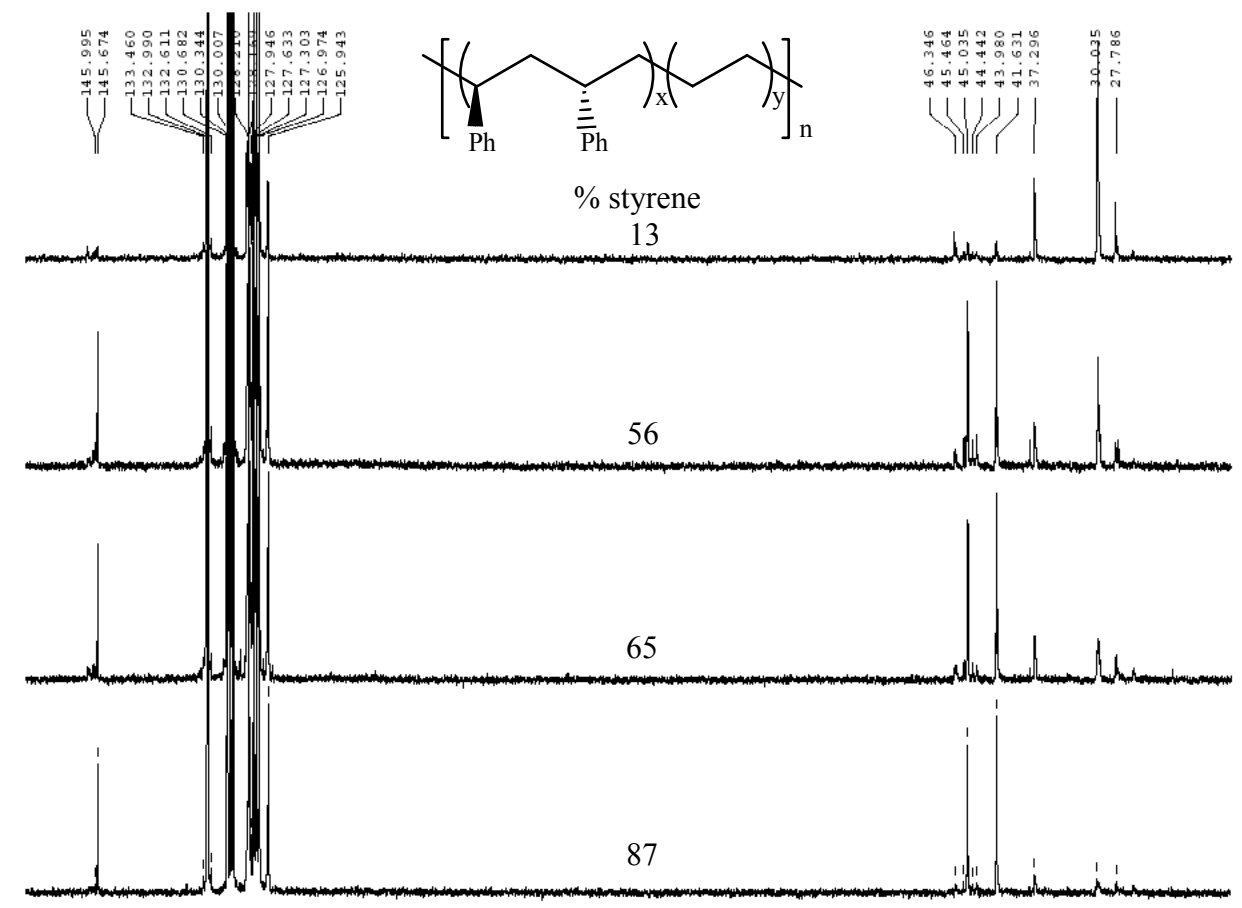

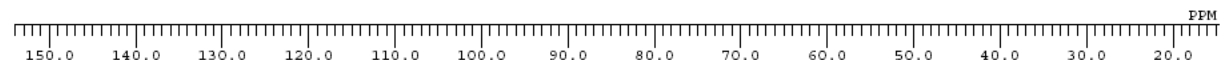

sFigure 2. ${ }^{13} \mathrm{C} \quad \mathrm{NMR}$ spectra of styrene-ethylene copolymers prepared from $\left(\mathrm{C}_{5} \mathrm{Me}_{4} \mathrm{SiMe}_{3}\right) \mathrm{Sc}\left(\mathrm{CH}_{2} \mathrm{SiMe}_{3}\right)_{2}(\mathrm{THF}) /\left[\mathrm{Ph}_{3} \mathrm{C}\right]\left[\mathrm{B}\left(\mathrm{C}_{6} \mathrm{~F}_{5}\right)_{4}\right]$ (runs 3-6, Table 2) in 1,2-dichlorobenzene- $d_{4}$ at $130{ }^{\circ} \mathrm{C}(75.5 \mathrm{MHz}$, HMDS scale). 

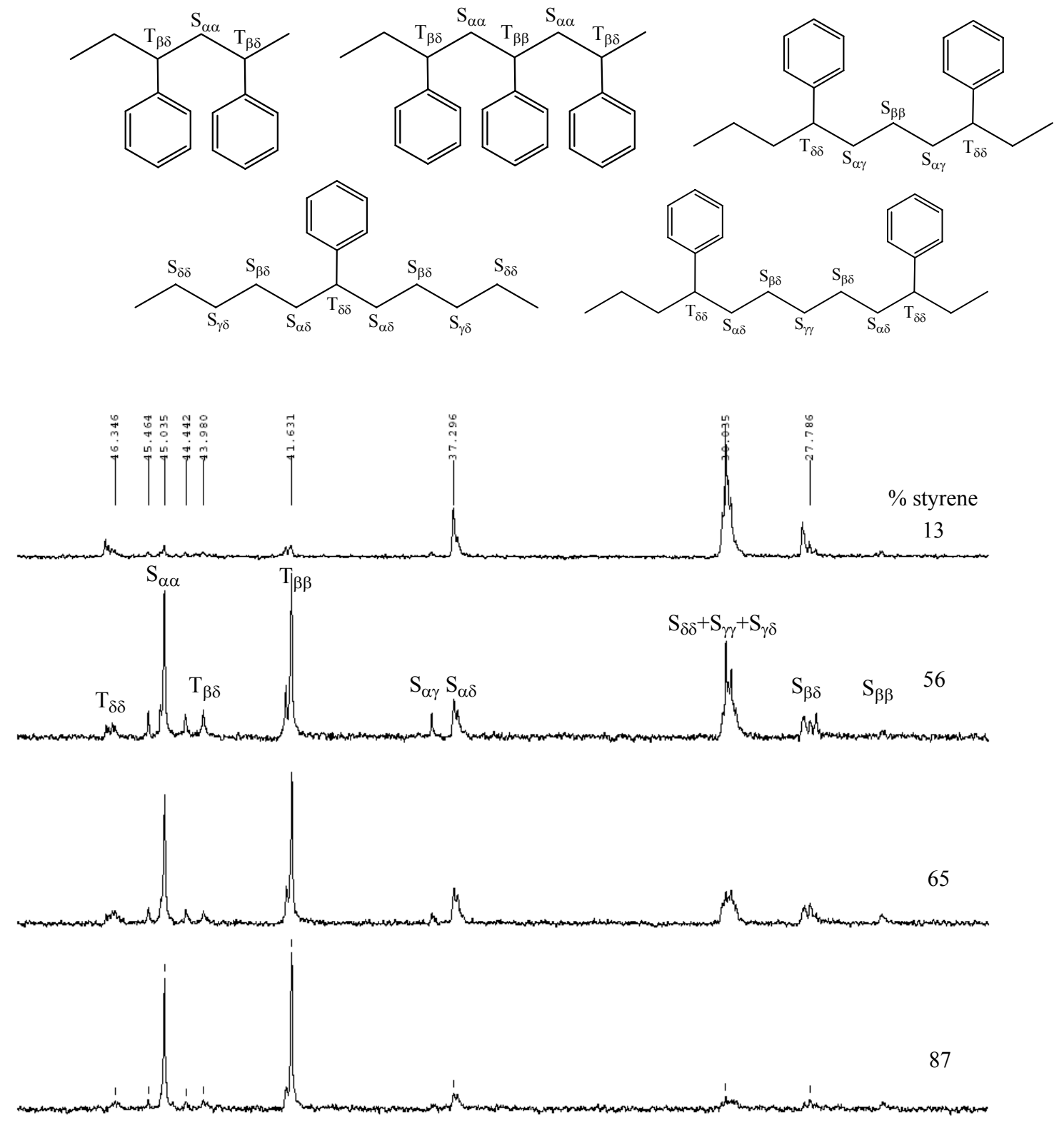

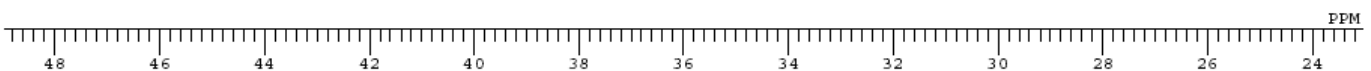

sFigure 3. Aliphatic region of ${ }^{13} \mathrm{C}$ NMR spectra of styrene-ethylene copolymers prepared from $\left(\mathrm{C}_{5} \mathrm{Me}_{4} \mathrm{SiMe}_{3}\right) \mathrm{Sc}\left(\mathrm{CH}_{2} \mathrm{SiMe}_{3}\right)_{2}(\mathrm{THF}) /\left[\mathrm{Ph}_{3} \mathrm{C}\right]\left[\mathrm{B}\left(\mathrm{C}_{6} \mathrm{~F}_{5}\right)_{4}\right]$ (runs 3-6, Table 2) in 1,2-dichlorobenzene- $d_{4}$ at $130{ }^{\circ} \mathrm{C}(75.5 \mathrm{MHz}$, HMDS scale). 


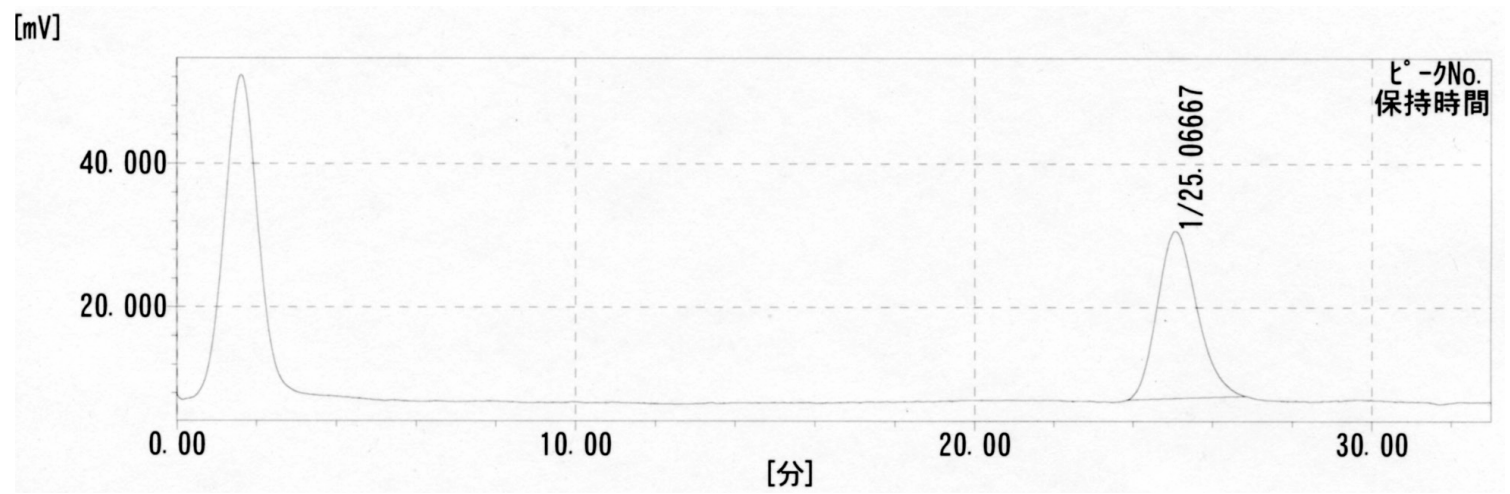

sFigure 4. GPC curve of styrene-ethylene copolymer prepared from $\left(\mathrm{C}_{5} \mathrm{Me}_{4} \mathrm{SiMe}_{3}\right) \mathrm{Sc}\left(\mathrm{CH}_{2} \mathrm{SiMe}_{3}\right)_{2}(\mathrm{THF}) /\left[\mathrm{Ph}_{3} \mathrm{C}\right]\left[\mathrm{B}\left(\mathrm{C}_{6} \mathrm{~F}_{5}\right)_{4}\right]$ (run 3, Table 2)

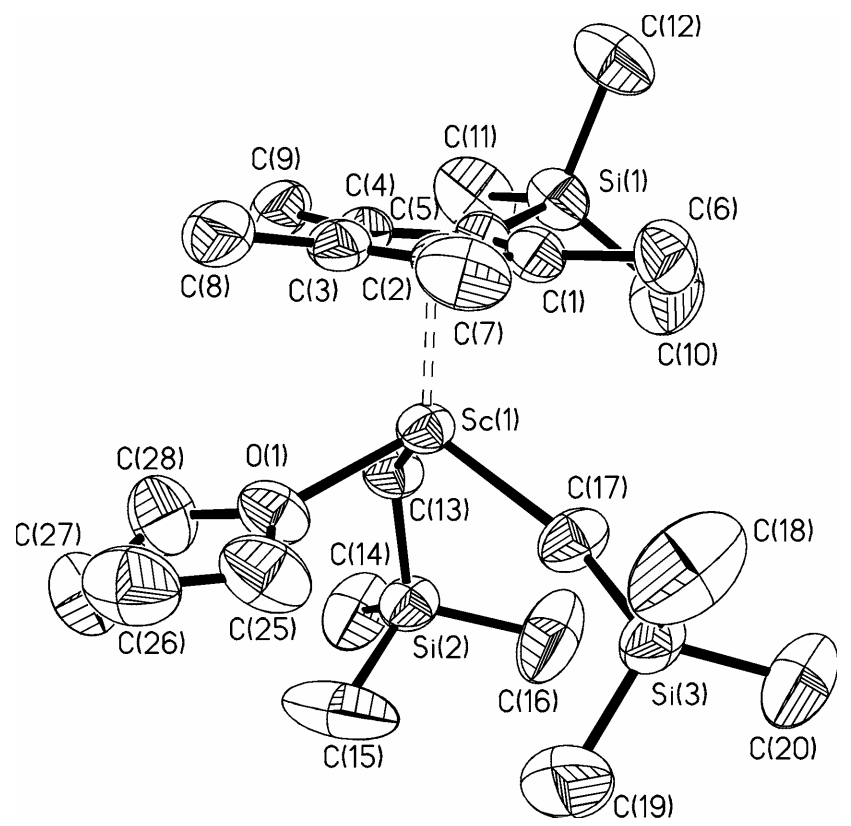

sFigure 5. ORTEP diagram of $\left(\mathrm{C}_{5} \mathrm{Me}_{4} \mathrm{SiMe}_{3}\right) \mathrm{Sc}\left(\mathrm{CH}_{2} \mathrm{SiMe}_{3}\right)_{2}(\mathrm{THF})$ with thermal ellipsoids at the $50 \%$ probability level. 


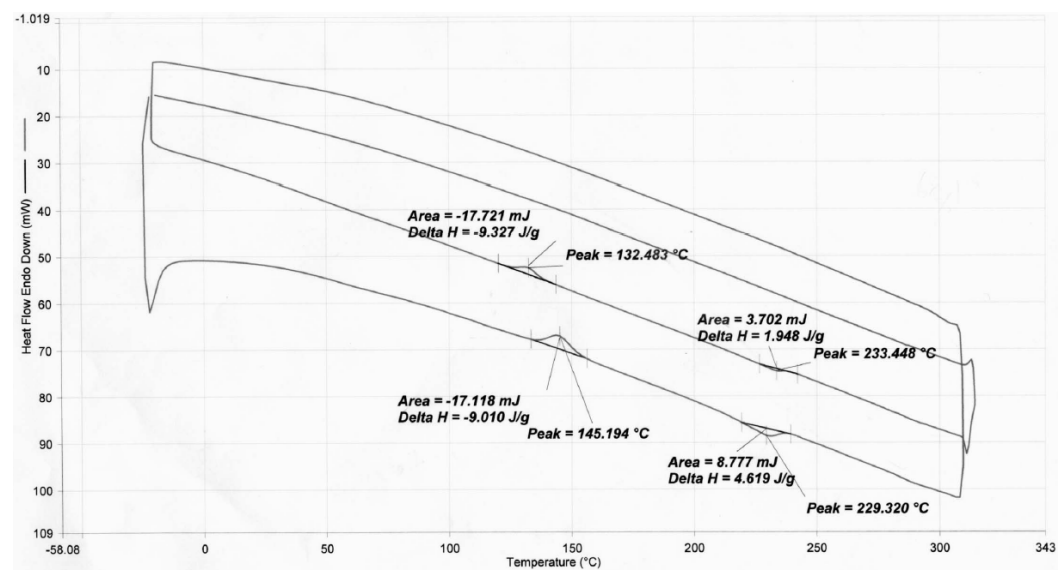

sFigure 6. DSC curve of styrene-ethylene copolymer (65\% styrene) prepared by use of $\left(\mathrm{C}_{5} \mathrm{Me}_{4} \mathrm{SiMe}_{3}\right) \mathrm{Sc}\left(\mathrm{CH}_{2} \mathrm{SiMe}_{3}\right)_{2}(\mathrm{THF}) /\left[\mathrm{Ph}_{3} \mathrm{C}\right]\left[\mathrm{B}\left(\mathrm{C}_{6} \mathrm{~F}_{5}\right)_{4}\right]$ (run 5, Table 2)

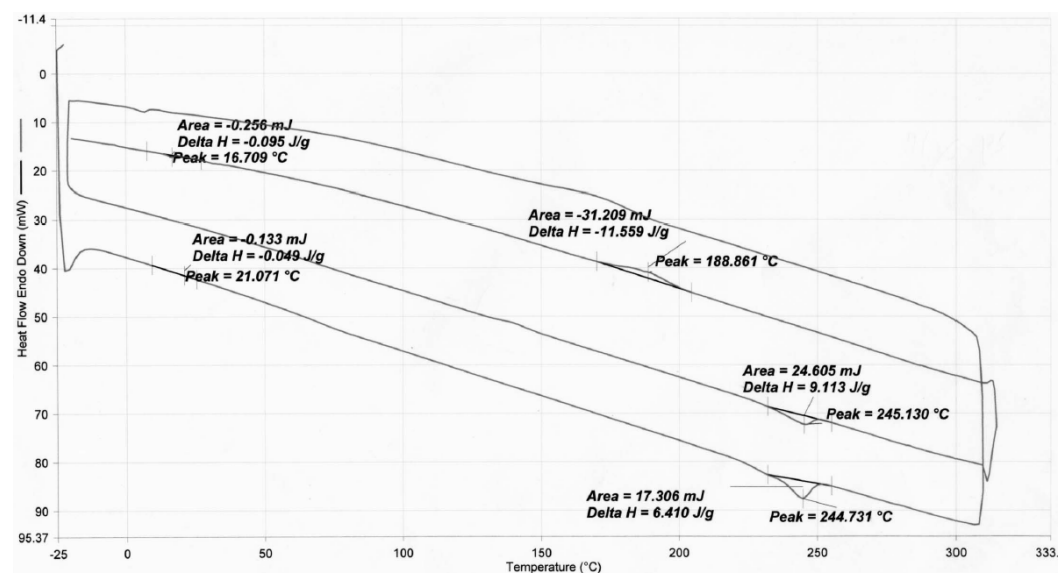

sFigure 7. DSC curve of styrene-ethylene copolymer ( $87 \%$ styrene) prepared by use of $\left(\mathrm{C}_{5} \mathrm{Me}_{4} \mathrm{SiMe}_{3}\right) \mathrm{Sc}\left(\mathrm{CH}_{2} \mathrm{SiMe}_{3}\right)_{2}(\mathrm{THF}) /\left[\mathrm{Ph}_{3} \mathrm{C}\right]\left[\mathrm{B}\left(\mathrm{C}_{6} \mathrm{~F}_{5}\right)_{4}\right]$ (run 6, Table 2) 